\title{
Antimicrobial and antioxidant activities of different extracts from Aspergillus unguis SPMD-EGY grown on different media
}

\author{
Ahmed A. Hamed ${ }^{1,2^{*}}$ (D), Mohamed S. Abdel-Aziz ${ }^{1,2}$ and Faten K. Abd El Hady ${ }^{3}$
}

\begin{abstract}
Background: Recently, researchers demonstrate the importance of associated microorganisms as potential sources of bioactive natural products. Especially, marine-derived fungi consider a treasure of new pharmaceutical leads due to the chemical diversity of their secondary metabolites.
\end{abstract}

Results: Aspergillus unguis SPMD-EGY was isolated from the soft coral Sinularia sp. and was cultivated on different media. Three broth media potato dextrose broth (PDB), peptone yeast extract malt extract glucose (PYMG), and Dox media in addition to one solid medium (rice medium) were used for this purpose. The fungus was grown under shake and static conditions (for broth media only). Ethyl acetate extract from both mycelia and culture supernatant and the extract from the direct extraction of rice solid medium were tested for their antimicrobial and antioxidant activities. It has been found that PYMG medium (mycelia static and culture static) exhibited the highest antimicrobial activity against all test microbes except Aspergillus niger followed by PDB medium (culture static and mycelia static). From the antioxidant point of view, the PDB medium showed the highest antioxidant activity (culture-shake) followed by Dox medium (culture static and mycelia static). The obtained results have been assessed and compared to the results of previous works constructed. GC/MS analysis of the fungal extracts showing high significant antimicrobial activities was evaluated.

Conclusion: Three different culture media were tested for the cultivation of the locally isolated fungus Aspergillus unguis isolate SPMD-EGY and were tested as antimicrobial and antioxidant agents. It has been found that growth media and growth incubation conditions have a very important role in secondary metabolites production.

Keywords: Aspergillus sp., Culture media, Antimicrobial, Antioxidant, DPPH

\section{Introduction}

As infectious diseases evolve to become more resistant to existing antibiotics, there is an undeniable need to discover new, safe, and effective drugs from natural sources with novel mechanisms of action to combat these pathogens. Marine environment is considered as an extremely diverse pool of life and has varied structurally unique biologically active natural products because of its extreme and rapidly changeable conditions (Zainuddin et al. 2010).

\footnotetext{
* Correspondence: ahmedshalbio@gmail.com; ahmed.shalabi@abdn.ac.uk ${ }^{1}$ Microbial Chemistry Department, National Research Centre, 33 El-Buhouth St., Dokki, P.O. 12622, Giza, Egypt

${ }^{2}$ Marine Biodiscovery Centre, Department of Chemistry, University of Aberdeen, Meston Walk, Aberdeen AB24 3UE, Scotland, UK

Full list of author information is available at the end of the article
}

The oceans are considered as a resource for discovery of different types of bioactive compounds such as nutritional material, enzymes, cosmetics, aflatoxins, and pharmaceuticals (Park et al. 2002; El-Shafei et al. 2010; El-Neekety et al. 2016). More than $70 \%$ of the Earth is covered by oceans, and it is believed that life on Earth originated from the sea (Haefner 2003). Although the terrestrial environment has been increasingly of interest as a source of new bioactive molecules, the marine environment is considered as a unique source of a diverse array of natural products, primarily from invertebrates such as tunicates, sponges, mollusks, and bryozoans, and from marine fungi, bacteria, and cyanobacteria.

Recently, the importance of marine organisms over the past $30-40$ years has been the focus of the researchers as potential sources for the discovery of chemotherapeutic 
agents (Kjer et al. 2010), more than 12,000 novel chemical structures with hundreds of new compounds still being discovered from marine plants, animals, and microbes every year (Donia and Hamann 2003). Additionally, a great percentage of marine microorganisms have not been described (Pomponi 1999). Marine associate microorganisms especially fungi were considered as an important and prolific source of biologically active metabolites (Saleem et al. 2007).

Many marine fungal strains were screened to assess their ability to produce antimicrobial, anticancer, and antiviral agents belonging to various compound classes including macrolides, terpenoids, alkaloids, or peptide derivatives (Bhadury et al. 2006). One of the most potent bioactive compound, the chlorinated benzophenone pestalone, was isolated from the marine fungus Pestalotia sp. and was shown to possess potent antibiotic activity against Staphylococcus aureus (MIC $=37 \mathrm{ng} / \mathrm{ml}$ ) and Enterococcus faecium ( $\mathrm{MIC}=78 \mathrm{ng} / \mathrm{ml}$ ), indicating that it could be evaluated further in advanced models of infectious disease (Cueto et al. 2001). Another new compound sorbicillactone A isolated from the sponge-derived fungus Penicillium chrysogenum exhibited promising activity against leukemia cells without exhibiting notable cytotoxicity (Zhou et al. 2018). This work has been undertaken with the aim of cultivation of the fungus on different medium in shaking and static conditions. The antimicrobial activity and the antioxidant activity, evaluated by two methods, will be investigated.

\section{Materials and methods}

\section{Fungal strain and culture condition}

The fungus Aspergillus unguis SPMD-EGY was isolated from the internal part from Sinularia sp. (soft coral) after cutting it into small cubes $(1 \mathrm{~cm})$ and using them to inoculate the isolation medium (PDA), and the fungus was previously identified by the molecular technique (18S rRNA) as previously described by Abd El-Hady et al. (2016a). Aspergillus sp. was cultivated on three broth media: potato dextrose broth (PDB), peptone yeast extract malt extract glucose (PYMG) broth, and Czapek-Dox (Dox) broth, in addition to the rice medium which is considered as solid medium (Abd El-Hady et al. 2016b).

\section{Extraction of secondary metabolites}

The fungi were harvested at the end of incubation period, centrifuged at $8000 \mathrm{rpm}$ and subjected to extraction. The culture supernatant was extracted with ethyl acetate $(\times 3$ or till exhaustion) and then evaporated under vacuum. On the other hand, the fungal mycelia were first extracted using acetone and evaporated till dryness. The residual part was re-extracted using a small volume of ethyl acetate (Abd El-Hady et al. 2014).

\section{Antimicrobial activity measurement}

Agar disc plate method has been established to measure the antimicrobial activities of different fungal extract samples (Collins and Lyne 1985; Youssef et al. 2014; Abdel-Aziz et al. 2015). Two bacterial test microbes: Staphylococcus aureus (Gram-positive) and Pseudononas aeruginosa (Gram-negative) and one yeast test microbe Candida albicans and one fungal test microbes, i.e. Aspergillus niger, were selected to evaluate the antimicrobial activities. The bacterial and yeast test microbes were grown on a nutrient agar medium (NA) of the following ingredients $(\mathrm{g} / \mathrm{l})$ : beef extract (3), peptone (10), and agar (20). On the other hand, the fungal test microbes were cultivated on Szapek-Dox agar medium of the following ingredients $(\mathrm{g} / \mathrm{l})$ : sucrose (30), $\mathrm{NaNO}_{3}$ (3), $\mathrm{MgSO}_{4} .7 \mathrm{H}_{2} \mathrm{O}(0.5), \mathrm{KCl}(0.5)$, $\mathrm{FeSO}_{4} .7 \mathrm{H}_{2} \mathrm{O}$ (0.055), $\mathrm{K}_{2} \mathrm{HPO}_{4}$ (1), and agar (20). The culture of each test microbe was diluted by distilled water (sterilized) to $10^{7}$ to $10^{8}$ colony-forming units $(\mathrm{CFU}) / \mathrm{ml}$ then $1 \mathrm{ml}$ of each was used to inoculate 11 Erlenmeyer flask containing $250 \mathrm{ml}$ of solidified agar media (Youssef et al. 2014). These media were put onto previously sterilized Petri dishes $(10 \mathrm{~cm}$ diameter having $25 \mathrm{ml}$ of solidified media). Filter paper discs $(5 \mathrm{~mm}$, Whatman No. 1 filter paper) were loaded with $0.2 \mathrm{mg}$ of each extract. The clay discs were placed on the surface agar plates seeded with test microbes and incubated for $24 \mathrm{~h}$ at the appropriate temperature of each test organism. Antimicrobial activities were recorded as the diameter of the clear zones (including the film itself) that appeared around the films (Abdel-Aziz et al. 2015).

\section{Antioxidant determination By DPPH method}

DPPH radical scavenging activity of all extracts was analyzed according to a modified procedure of Matsushige and his group (Matsushige et al. 1996). One milliliter of methanol solution for each extract $(100 \mu \mathrm{g} / \mathrm{ml})$ was added to $1 \mathrm{ml}$ of methanol solution of DPPH $(60 \mu \mathrm{M})$. The prepared solutions were mixed and left for $30 \mathrm{~min}$ at room temperature. The optical density was measured at $520 \mathrm{~nm}$ using a spectrophotometer (UV-1650PC Shimadzu, Japan). Mean of three measurements for each compound was calculated. Caffeic acid was used as a positive control with $80.2 \%$ scavenging activity.

\section{By superoxide anion scavenging activity (XOD)}

Superoxide anion scavenging activity was determined according to a modified method of Matsushige and his group (Matsushige et al. 1996). Reaction mixtures containing $1.4 \mathrm{ml}$ of $50 \mathrm{mM} \mathrm{Na}_{2} \mathrm{CO}_{3}(\mathrm{pH} \mathrm{10.2),} 100 \mu \mathrm{l}$ of 3 $\mathrm{mM}$ xanthine, $100 \mu \mathrm{l}$ of $3 \mathrm{mM}$ EDTA, $100 \mu \mathrm{l}$ of BSA (1.5 $\mathrm{mg} / \mathrm{ml}), 100 \mu \mathrm{l}$ of $75 \mathrm{mM}$ Nitro blue tetrazolium, and $50 \mu \mathrm{l}$ of each compound $(100 \mu \mathrm{g} / \mathrm{ml})$ were preincubated at $30{ }^{\circ} \mathrm{C}$ for $10 \mathrm{~min}$, and $50 \mu \mathrm{l}$ of xanthine oxidase $(0.3$ 
unit/ml) was added. After incubation at $30{ }^{\circ} \mathrm{C}$ for 20 min, $200 \mu \mathrm{l}$ of $6 \mathrm{mM} \mathrm{CuCl}_{2}$ was added to stop the reactions, and the absorbance was measured at $560 \mathrm{~nm}$. Caffeic acid was used as a positive control with $73.5 \%$ scavenging activity.

\section{GC/MS analyses}

One milligram of the dried extract was prepared for chromatography by derivatization for $30 \mathrm{~min}$ at $85^{\circ} \mathrm{C}$ with $20 \mu \mathrm{l}$ pyridine $+30 \mu \mathrm{l} \mathrm{N}, \mathrm{O}$, bis-(trimethylsilyl)trifluoroacetamide (BSTFA) and analyzed by GC/MS (Abd El-Hady et al. 2016a). A Finnigan MAT SSQ 7000 mass spectrometer was coupled with a Varian 3400 gas chromatograph. DB-1 column, $30 \mathrm{~m} \times 0.32 \mathrm{~mm}$ (internal diameter), was employed with helium as carrier gas (He pressure, $20 \mathrm{Mpa} /$ $\mathrm{cm}^{2}$ ), injector temperature, $310^{\circ} \mathrm{C}$; and $\mathrm{GC}$ temperature program, $85-310^{\circ} \mathrm{C}$ at $3{ }^{\circ} \mathrm{C} / \mathrm{min}$ (10 min initial hold). The mass spectra were recorded in electron ionization (EI) mode at $70 \mathrm{eV}$. The scan repetition rate was $0.5 \mathrm{~s}$ over a mass range of 39-650 amu.

\section{Statistical analysis}

Data were presented as mean \pm standard deviation of triplicates $(n=3)$ according to Annegowda et al. 2010 using SPSS 13.0 program (SPSS Inc. USA) (Annegowda et al. 2010).

\section{Results}

Ethyl acetate extracts from the fungus SPMD, previously identified as Aspergillus unguis isolate SPMD-EGY (accession no. KM203833) and was cultivated on different culture media, i.e., potato dextrose broth (PDB), peptone yeast extract malt extract glucose (PYMG), Czapek-Dox (Dox), and rice (solid) media, were evaluated for their antimicrobial activities. It has been found that the antimicrobial activities were higher when the fungus was grown on PYMG broth medium (culture static and mycelia static) against Staphylococcus aureus, Pseudomonas aeruginosa, and Candida albicans. Potato dextrose broth (culture static and mycelia static) exhibited slightly higher antimicrobial activities against all tested microbes (Fig. 1). All the produced extracts from the different medium by this fungus (Aspergillus unguis SPMD-EGY) did not exhibit any antifungal activity against Aspergillus niger (test microbe). Results in Fig. 2 distinguish the antimicrobial activity of our previous work of the same fungus grown on the same broth medium with the only differences are the utilization of different glucose concentration and seawater (Abd El-Hady et al. 2016a). It has been found that the produced extracts exhibited nearly the same results with the only difference is that there is an antimicrobial activity against the fungus Aspergillus niger. Results in Fig. 3 revealed that the antioxidant activity from Aspergillus unguis SPMD-EGY grown on potato dextrose broth (PDB) medium exhibited the promising activity measured by both DPPH and X-XOD under shake conditions from culture supernatant ethyl acetate extract. Also, PDB exhibited reasonable antioxidant activity (DPPH) under shake and static conditions from
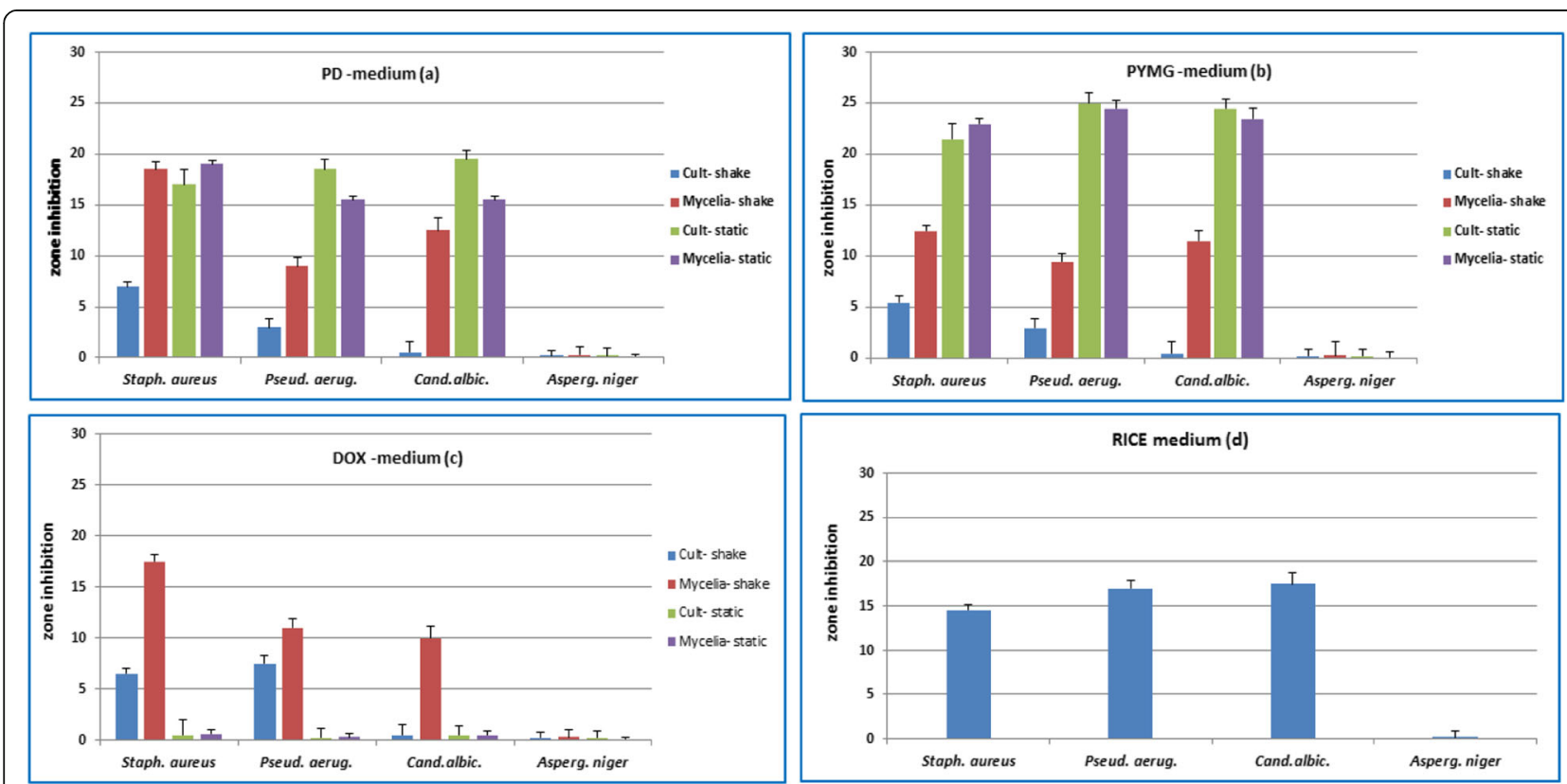

Fig. 1 Antimicrobial activity of secondary metabolite extracts from culture and mycelia (static and shake) of different media. Values are expressed as mean \pm SD, $n=3(200 \mu \mathrm{g} / \mathrm{ml}$ for all tested extracts 

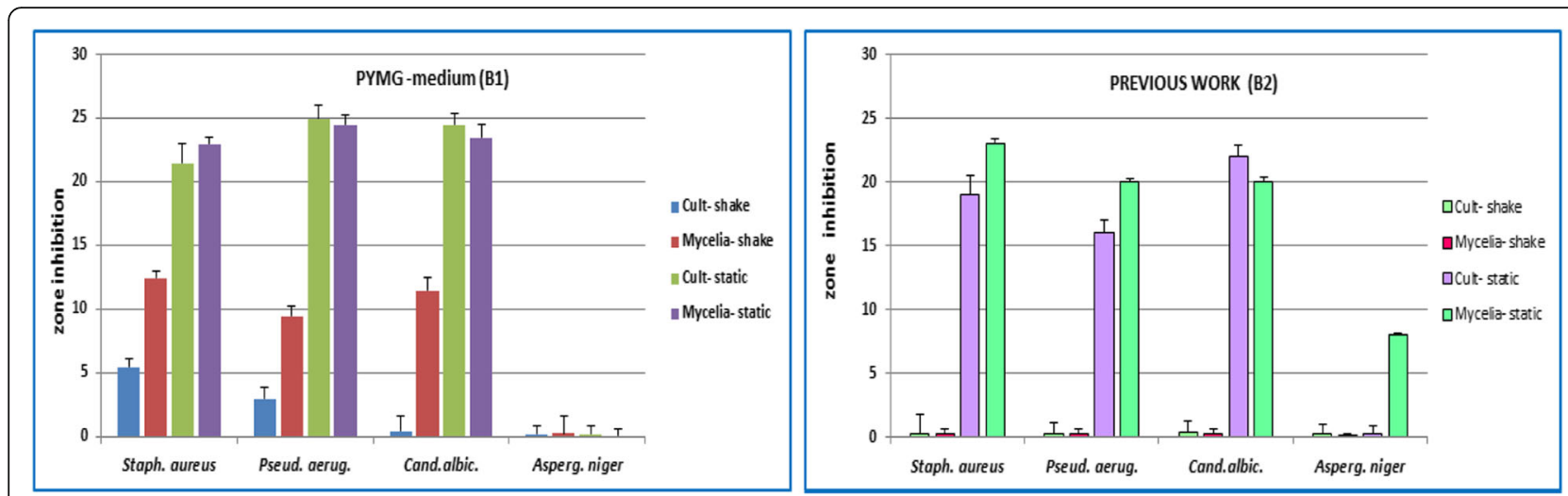

Fig. 2 Demonstrative antimicrobial comparison between PYMG medium (5\% glucose, distilled water) and previous work medium (10\% glucose, seawater)

culture supernatant and mycelia extracts, respectively, Czapek-Dox (Dox) broth medium followed PDB medium for antioxidant activity. It has been investigated that the DPPH and X-XOD activities were noticed under static conditions for both culture supernatant and mycelia ethyl acetate extracts. On the other hand, PYMG broth medium and rice solid medium showed restricted antioxidant activities (for DPPH only). The free radical scavenging activities measured by DPPH and X-XOD were compared to that have been previously done (Abd El-Hady et al. 2016a) and illustrated in Fig. 4. It has been found that the scavenging activities measured by DPPH showed approximately the same results, but X-XOD exhibited the highest differences for both.

The GC/MS analysis for fungal extracts showing highly significant antimicrobial activities (mycelia static and culture static of PYMG and PD media) was done. A total of 63 compounds were identified. Table 1 revealed the complete variation in the chemical composition of the four fungal extracts.

Hexadecanoic acid (18\%), 9,12-Octadecadienoic acid, 9-Octadecenoic acid, 8-Methoxy-2,3-dihydro-1 h-benzazepin-2-one-5-acetic acid, and diisooctyl phthalate were
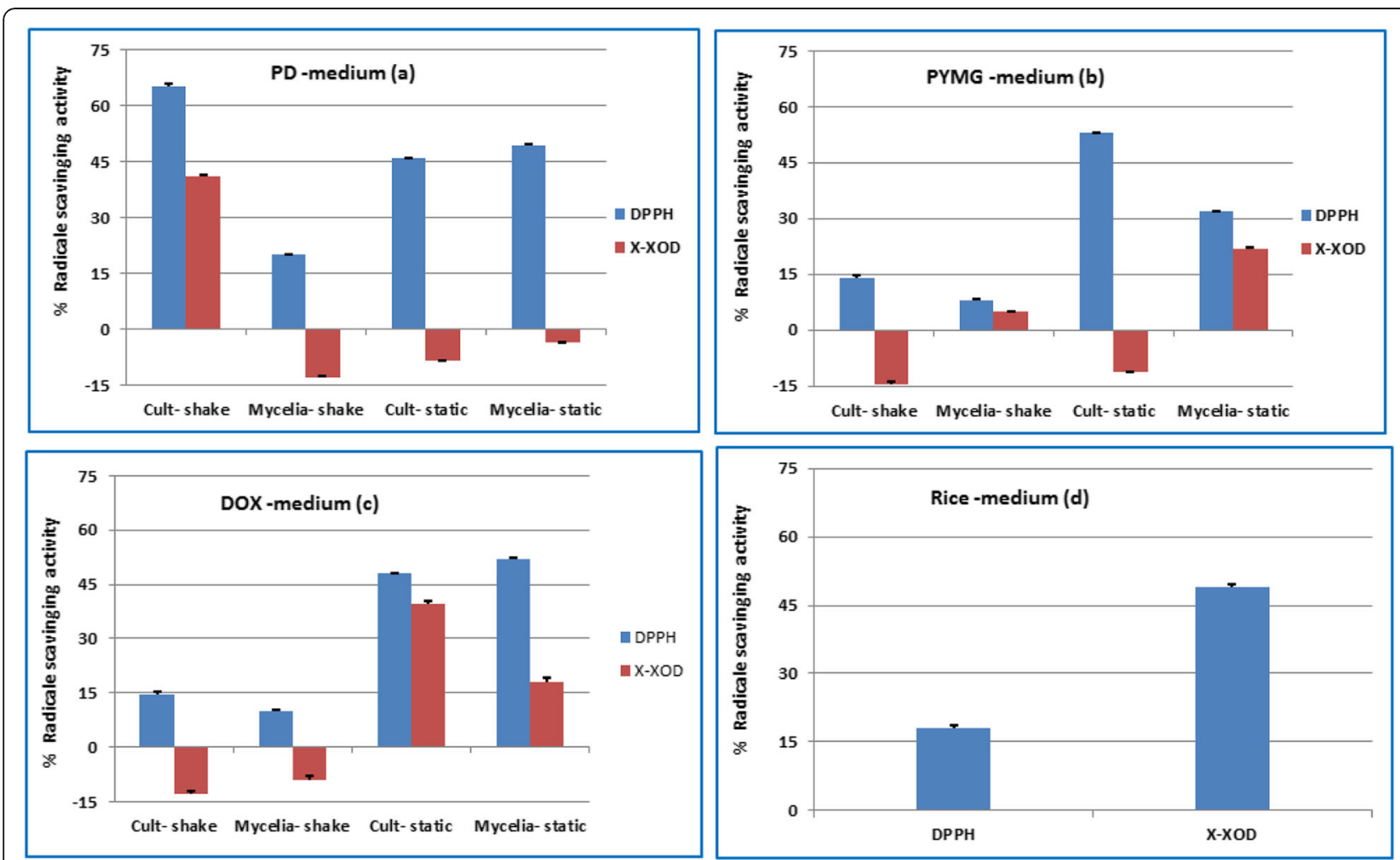

Fig. 3 Free radical scavenging activity of secondary metabolite extracts from culture and mycelia (static and shake) of different media in the $\mathrm{DPPH}$ radical and xanthine-XOD assays. Values are expressed as mean $\pm \mathrm{SD}, n=3$, at a concentration of $200 \mu \mathrm{g} / \mathrm{ml}$ for all tested extracts 

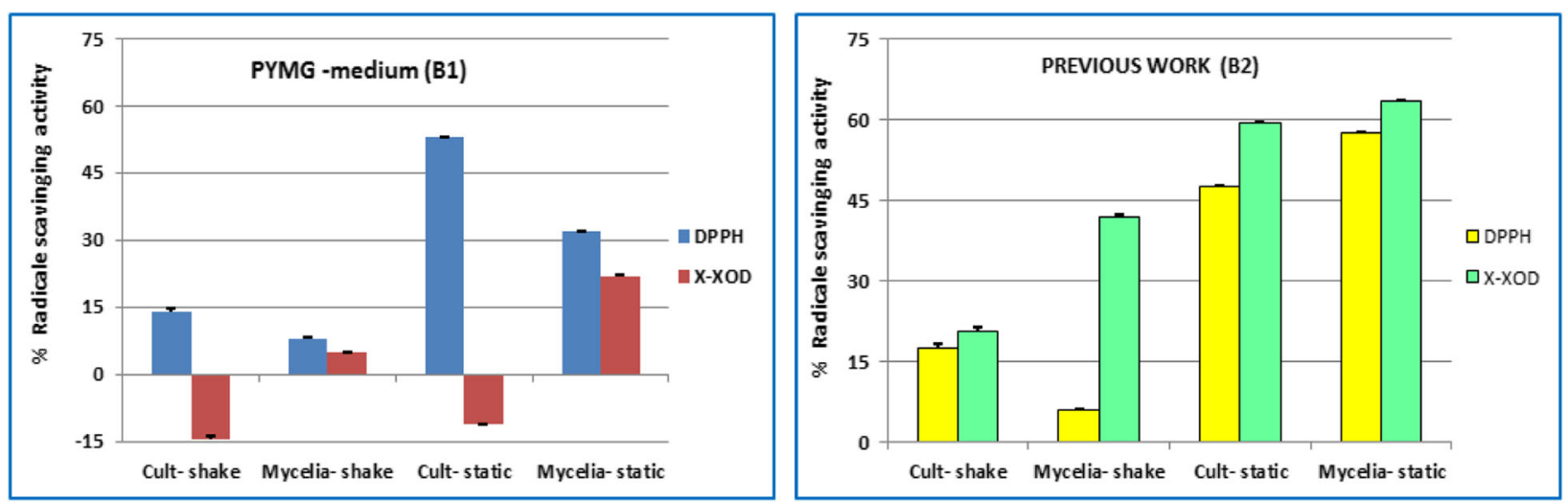

Fig. 4 Demonstrative antioxidant comparison between PYMG medium (5\% glucose, distilled water) and previous work medium (10\% glucose, seawater)

present in PYMG mycelia static (M.st.) extract with high concentrations. 2,2'-Dihydroxy-chalcone (33.6\%), D-Friedoolean-14-en-3-one (39.8\%), 1,6,7-Trimethyl-3-phenyl9H-xanthen-9-one, and 2,4,4'-Trihydroxy-chalcone were present in PYMG culture static (C.st) extract with high concentration. Dodecanoic acid and 2,4-Dihydroxybenzoic acid were mainly found in PD mycelia static (M.st) extract, while in its culture static (C.st) extract, hexadecanoic acid (11.6\%) and Acetamide-2,2,2-trifluoro (19.3\%) were present in high concentrations.

\section{Discussion}

Growth media and growth incubation conditions have a strong effect on secondary metabolite production, and there is no compromise on which culture media are optimal for specific metabolite production (Mathan et al. 2013). Several culture media like Czapek-Dox broth, Sabourod broth, potato dextrose broth, malt extract broth, and nutrient broth were used to investigate the antimicrobial activities from Aspergillus terreus KC582297. Potato dextrose broth was found to be a potent medium for the production of bioactive antimicrobial metabolites (Mathan et al. 2013). Noaman et al. (2004) studied the effect of different concentrations of glucose on growth and antimicrobial activity from Synochococcus leopoliensis. It has been found that maximum antimicrobial activity was reported at a glucose concentration of $0.6 \mathrm{~g} / \mathrm{l}$. On the other hand, antimicrobial activity from the fungus Arthrinium c.f. saccharicola was higher after cultivating it on medium with higher glucose concentration (Miao et al. 2006). The bioactivity from this fungus was also increased with increasing salinity (up to $3 \mathrm{ppm}$ ). It has been reported that the antioxidant activity is species- and culture medium-dependent (Dulay et al. 2015a). Antioxidant activities from Penicillium antillarium grown on different broth media exhibited the highest scavenging activity with potato broth medium (Dulay et al. 2015b). Moreover, ethyl acetate extract from different broth media including rice bran, coconut water, corn grit, and potato broth and all cultivated with Lentinus spp. were tested for their antioxidant activities. It has been found that rice bran exhibited the highest scavenging activities for both Lentinus spp. (Dulay et al. 2015a). Ethyl acetate extract from Czapek-Dox medium cultivated with Chaetomium madrasense exhibited the highest antioxidant activity (Abo-Elmagd 2015).

It was reported that free fatty acids showed antimicrobial activities against 12 oral pathogens which cause dental caries, stomatitis, periodontitis, and gingivitis (Choi et al. 2013). Long-chain unsaturated fatty acids, such as linoleic acid, show antibacterial activity. Additional unsaturated fatty acids including palmitoleic acid, oleic acid, linolenic acid, and arachidonic acid also exhibited antibacterial activity (Zheng et al. 2005). Lauric, palmitic, linolenic, linoleic, oleic, stearic, and myristic acids are known to have potential antibacterial and antifungal agents (Agoramoorthy et al. 2007). Investigation of in vitro synergism between several chalcones substituted in combination with oxacillin, an antibiotic used conventionally against $S$. aureus ATCC 43300 that is resistant to meticillin, using the kinetic turbidimetric method developed earlier. The results were satisfactory for all assayed combinations and in accordance with the mechanism of bacteriostatic inhibition previously proposed, except for $2^{\prime}, 4^{\prime}$-dihydroxy-3' -methoxychalcone - oxacillin (Talia et al. 2011).

D-Friedoolean-14-en-3-one was demonstrated in vitro antileishmanial and antitumor activities (Moulisha et al. 2009). 2,4-Dihydroxybenzoic and protocatechuic acids were the phenolic compounds with higher activity against the majority of Gram-negative and Gram-positive bacteria. Furthermore, phenolic compounds inhibited more MRSA than methicillin-susceptible Staphylococcus aureus. MRSA was inhibited by 2,4-dihydroxybenzoic, vanillic, syringic acids. The presence of carboxylic acid $(\mathrm{COOH})$, two hydroxyl $(\mathrm{OH})$ groups in para- and ortho positions of the 
Table 1 Chemical composition assessed by GC/MS analysis for fungal extracts with high antimicrobial activities from different media for Aspergillus unguis SPMD-EGY

\begin{tabular}{|c|c|c|c|c|c|}
\hline \multirow[t]{3}{*}{ No. } & \multirow[t]{3}{*}{ Compound } & \multicolumn{2}{|c|}{ PYMG medium } & \multicolumn{2}{|c|}{ PD medium } \\
\hline & & $\overline{\text { M. st. }^{a}}$ & CC. st. ${ }^{b}$ & M. st. ${ }^{c}$ & CC. st. \\
\hline & & \multicolumn{4}{|l|}{${ }^{*} \mathrm{TIC} \%$} \\
\hline & \multicolumn{5}{|l|}{ Saturated fatty acids } \\
\hline 1 & Butanedioic acid & 1.0 & & & \\
\hline 2 & Butanoic acid-2-methyl & & & 0.78 & \\
\hline 3 & Butanoic acid-4-hydroxy & & & 0.05 & \\
\hline 4 & Azelaic acid & 0.8 & & & \\
\hline 5 & Propanoic acid, 2,3-dihydroxy & & & 0.22 & \\
\hline 6 & 2-Isopropyl-2-hydroxy succinic acid & & & 0.04 & \\
\hline 7 & Dodecanoic acid (lauric acid) & & & 7.13 & \\
\hline 8 & Tetradecanoic acid (myristic acid) & 0.4 & & & \\
\hline 9 & Pentadecanoic acid & 0.4 & & & \\
\hline 10 & Hexadecanoic acid (palmitic) & 18.0 & & 1.63 & 11.64 \\
\hline 11 & Heptadecanoic acid & 0.3 & & & \\
\hline \multirow[t]{2}{*}{12} & Octadecanoic acid (stearic acid) & 3.1 & & 0.41 & 0.73 \\
\hline & \multicolumn{5}{|l|}{ Unsaturated fatty acids } \\
\hline 13 & Cis-9-hexadecenoic acid (oleic acid) & 0.6 & & & \\
\hline 14 & Heptadecenoic acid & 0.3 & & & \\
\hline 15 & 9,12-Octadecadienoic acid (linoleic acid) & 3.4 & & & \\
\hline 16 & 9-Octadecenoic acid & 3.5 & & 1.11 & 0.96 \\
\hline \multirow[t]{2}{*}{17} & 11-Octadecenoic acid & 0.3 & & & \\
\hline & \multicolumn{5}{|l|}{ Fatty acid esters } \\
\hline 18 & 5-Keto-2,2-dimethyl-heptanoic acid, propyl ester & 0.6 & & & \\
\hline 19 & Hexadecanoic acid-2,3-dihydroxy propyl ester & 0.6 & & & \\
\hline 20 & 9,12-Octadecadienoic acid-2-hydroxy-ethyl ester & 0.3 & & & \\
\hline 21 & Octadecanoic acid-2,3-dihydroxy- propyl ester & & & 0.16 & \\
\hline \multirow[t]{2}{*}{22} & Docosanoic acid,1,2,3-propanetriyl ester & & & 0.11 & \\
\hline & \multicolumn{5}{|l|}{ Phenolic acids } \\
\hline 23 & Benzoic acid & & & 0.14 & \\
\hline 24 & Benzeneacetic acid-a-4-dihydroxy & & & 0.13 & \\
\hline 25 & Benzeneacetic acid-2-hydroxy & & & 0.17 & \\
\hline 26 & Benzenepropanoic acid-a-hydroxy & & & 0.09 & \\
\hline 27 & 2,4-Dihydroxybenzoic acid & & & 2.65 & \\
\hline \multirow[t]{2}{*}{28} & Caffeic acid & & & 0.64 & \\
\hline & \multicolumn{5}{|l|}{ Nitrogenous compounds } \\
\hline 29 & 8-Methoxy-2,3-dihydro-1 h-benzazepin-2-one-5-acetic acid & 6.2 & & & \\
\hline 30 & Acetamide-2,2,2-trifluoro & & & & 19.3 \\
\hline 31 & Morpholine & & & & 2.7 \\
\hline 32 & $\mathrm{~N}, \mathrm{~N}$-diethylamine & & & & 0.20 \\
\hline 33 & $N$-Acetyl- $N$-methyl alanine & 3.1 & & & \\
\hline 34 & $\mathrm{~N}$-I-valyl-I-valinate & & & 0.12 & \\
\hline 35 & 3-Pyridine-carboxylic acid & & & 0.1 & \\
\hline 36 & 2,6-Ditbutyl-4-hydroxy-4-phenyl-1-imino-2,5-cyclohexadiene & 0.4 & & & \\
\hline
\end{tabular}


Table 1 Chemical composition assessed by GC/MS analysis for fungal extracts with high antimicrobial activities from different media for Aspergillus unguis SPMD-EGY (Continued)

\begin{tabular}{|c|c|c|c|c|c|}
\hline \multirow[t]{3}{*}{ No. } & \multirow[t]{3}{*}{ Compound } & \multicolumn{2}{|c|}{ PYMG medium } & \multicolumn{2}{|c|}{ PD medium } \\
\hline & & $\overline{\text { M. st. }{ }^{a}}$ & CC. st. ${ }^{\mathrm{b}}$ & M. st. ${ }^{\mathrm{C}}$ & CC. st. ${ }^{\mathrm{d}}$ \\
\hline & & \multicolumn{4}{|l|}{${ }^{*} \mathrm{TIC} \%$} \\
\hline 37 & 4(4-Methoxyphenyl)-4-(1H-pyrrolo[2,3b]pyridin-3-yl)butan-2-one & 0.8 & & & \\
\hline 38 & 7,8-Dihydroxy-benzo(5,6 g)1H,3H-quinazoline-2,4-dione & 0.7 & & & \\
\hline 39 & 1-Imino6(4-methylphenyl)3-oxo-2,5dipheny-11,2,3,5,6-pentahydro-imidazo[1,5a]pyrazine-8-carboxamide & & & 2.5 & \\
\hline 40 & 4,6-Dimethoxy-7(5-methyl-1-pyrrolin-2-yl)2,3-diphenylindole & 0.22 & & & \\
\hline 41 & 2,2-Dichloro-1,1-bis(4-diethylaminophenyl)ethylene & 0.8 & & & \\
\hline \multirow[t]{2}{*}{42} & 1,1,3-Trifluoro-3,3-bis[ethylamino]1-phenyldisiloxane & 3.5 & & & \\
\hline & Others & & & & \\
\hline 43 & Glycerol & 2.3 & & & \\
\hline 44 & Methyl -7(2,4,6-trimethylphenyl)-5H-furo[2,3c] thiopyran-4-carboxylate & 0.5 & & & \\
\hline 45 & Tridecanol & 0.62 & & & \\
\hline 46 & L-Threitol & 2.0 & & & \\
\hline 47 & 6(4 t-Butylphenyl)1,3,5-hexatriynyl)trimethylsilane & 1.5 & & & \\
\hline 48 & Arabitol & 3.4 & & & \\
\hline 49 & 1,1,4,4-Tetramethyl-2,3-diphenyl-1,4-disilacyclohex-2ene & 0.93 & & & \\
\hline 50 & 1,1,4,4Tetramethyl2,3diphenyl1,4di-silacyclohex2ene (isomer) & 0.89 & & & \\
\hline 51 & 4,4'-Dihydroxy-chalcone & 1.3 & & & \\
\hline 52 & 3-Deoxyglucitol & 0.45 & & & \\
\hline 53 & Sorbitol & 6.1 & & & \\
\hline 54 & 2,2'-Dihydroxy-chalcone & & 33.6 & & \\
\hline 55 & Methyl $2\{2[3$ (methoxycarbonyl)phenyl]-1-ethyny| $\} 5$-hydroxy-ethynyl)benzoate & 0.8 & & & \\
\hline 56 & Erythrose & & & 0.07 & \\
\hline 57 & Glucose oxime & & & & 0.50 \\
\hline 58 & D-Gluconic acid & & & & 2.45 \\
\hline 59 & D-Galactonic acid-a-lactone & & & 0.11 & \\
\hline 60 & D-Friedoolean-14-en-3-one & & 39.8 & & \\
\hline 61 & 1,6,7-Trimethyl-3-phenyl-9H-xanthen-9-one and & & 3.1 & & \\
\hline 62 & $2,4,4^{\prime}$-Trihydroxy-chalcone & & 3.2 & & \\
\hline 63 & Diisooctyl phthalate & 13.8 & & 0.16 & 0.30 \\
\hline
\end{tabular}

*TIC the ion current generated depends on the characteristics of the compound concerned and it is not a true quantitation, $M$. st. mycelia static, C. st. culture static

${ }^{a}$ Data previously mentioned (Abd El-Hady et al. 2016a); b,d(Abd El-Hady et al., 2016c); (Abd El-Hady et al. 2016d)

benzene ring and also a methoxyl (OCH3) group in the meta position seems to be important for anti-MRSA activity (Alves et al. 2013). N-benzyl-2,2,2-trifluoroacetamide was assessed for its antimicrobial and antioxidant. It showed good antifungal activity against tested fungi, specially C. albicans and moderate antibacterial activity. $N$-benzyl-2,2,2-trifluoroacetamide showed high antioxidant activity. Cupric ion reducing the antioxidant capacity of $N$-benzyl-2,2,2-trifluoroacetamide was dependent on the concentration (Balachandran et al. 2015). The antibacterial and antifungal activities of ethyl acetate extract, di-(2-ethylhexyl) phthalate (compound 1) and compound 2 were measured using the disc diffusion method. Ethyl acetate extract and compound 1 presented better results than anhydrosophoradiol-3-acetate (compound 2).

\section{Conclusion}

The utilization of different growth media exhibited a prolific role in the production of unique bioactive secondary metabolites with a unique chemical skeleton. Three different culture media, namely potato dextrose broth (PDB), peptone yeast extract malt extract glucose (PYMG), and Czapek-Dox (Dox) and rice (solid) media were used for the cultivation of the locally isolated fungus Aspergillus unguis isolate SPMD-EGY (accession no. KM203833), and the produced ethyl acetate extract from 
each medium were tested as antimicrobial and antioxidant agents. Diverse results have been obtained and varied from medium to another medium.

\section{Acknowledgement}

This work is financially supported by the bilateral projects within the Executive Program of Scientific and Technological Cooperation between the Arab Republic of Egypt and Italian Rep. for the years 2013-2018. Project no. [A2-12-15].

\section{Funding}

This work was supported by the Ministry of Higher Education and Scientific Research, Egypt (MHESR).

\section{Availability of data and materials}

Data are available on request from the authors.

\section{Authors' contributions}

MA-A, FAEH, and $\mathrm{AH}$ contributed to the design and implementation of the research, analysis of the results, writing of the manuscript. $\mathrm{FAEH}, \mathrm{AH}$, and MAA performed the experiments. All authors read and approved the final manuscript.

\section{Ethics approval and consent to participate}

Not applicable

\section{Consent for publication}

Not applicable

\section{Competing interests}

The authors declare that they have no competing interests.

\section{Publisher's Note}

Springer Nature remains neutral with regard to jurisdictional claims in published maps and institutional affiliations.

\section{Author details}

${ }^{1}$ Microbial Chemistry Department, National Research Centre, 33 El-Buhouth St., Dokki, P.O. 12622, Giza, Egypt. ${ }^{2}$ Marine Biodiscovery Centre, Department of Chemistry, University of Aberdeen, Meston Walk, Aberdeen AB24 3UE, Scotland, UK. ${ }^{3}$ Chemistry of Natural Products, National Research Center, Dokki, Giza, Egypt.

Received: 1 August 2018 Accepted: 6 November 2018 Published online: 03 December 2018

\section{References}

Abd El-Hady FK, Abdel-Aziz MS, Shaker KH, El-Shahid ZA (2016a) Effect of media composition on potentiality improving a-glucosidase inhibitory activity for the soft coral associated fungus Aspergillus unguis SPMD-EGY. Int J Pharm Sci Rev Res 38(2):173-180

Abd El-Hady FK, Abdel-Aziz MS, Shaker KH, El-Shahid ZA, Ghani MA (2014) Coral derived fungi inhibit acetyl cholinesterase, superoxide anion radical, and microbial activities. Int J Pharm Sci Rev Res 26(1):301-308

Abd El-Hady FK, Abdel-Aziz MS, Souleman AMA, El-Shahid ZA, Shaker KH (2016c) Enhancement of Acetylcholinesterase Inhibitory Activity for the Soft Coral Associated Fungus Aspergillus unguis SPMD-EGY by Media Composition. Int J Pharm Sci Rev Res 41:349-357

Abd El-Hady FK, Abdel-Aziz MS, Souleman AMA, El-Shahid ZA, Shaker KH (2016d) Potentiality of Improving or Suppressing Tyrosinase Inhibitory Activity by Media Composition for the Marine Fungus Aspergillus unguis SPMD-EGY. Der PharmaChemica 8(19):458-465

Abd El-Hady FK, Souleman AMA, Ibrahim IG, Abdel-Aziz MS, El-Shahid ZA, Ali EA, Elsarrag MSA (2016b) Cytotoxic, anti-acetylcholinesterase, antioxidant and antimicrobial activities of Sudanese propolis with correlation to its GC/MS and HPLC analysis. Pharm Lett 8(19):339-350

Abdel-Aziz MS, Abou-El-Sherbini KS, Hamzawy EMA, Amr MHA, El-Dafrawy S (2015) Green synthesis of silver nanoparticles by Macrococcus bovicus and its immobilization onto montmorillonite clay for antimicrobial functionality. Appl Biochem Biotechnol 176:2225-2241
Abo-Elmagd HI (2015) Evaluation and optimization of antioxidant potentiality of Chaetomium madrasense AUMC 9376. J Genet Eng Biotechnol 12(1):21-26

Agoramoorthy G, Chandrasekaran M, Venkatesalu V, Hsu MJ (2007) Antibacterial and antifungal activities of fatty acid methyl esters of the blind-your-eye mangrove from India. Braz J Microbiol 38(4):739-742

Alves MJ, Ferreira IC, Froufe HJ, Abreu RM, Martins A, Pintado M (2013) Antimicrobial activity of phenolic compounds identified in wild mushrooms, SAR analysis and docking studies. J Appl Microbiol 115:346-357

Annegowda HV, Nee CW, Mordi MN, Ramanathan S, Mansor SM (2010) Evaluation of phenolic content and antioxidant property of hydrolysed extracts of Terminalia catappa (L.) leaf. Asian J Plant Sci 9:479-485

Balachandran C, Kumar PS, Arun Y, Duraipandiyan V, Sundaram RL, Vijayakumar A, Balakrishna K, Ignacimuthu S, Al-Dhabi NA, Perumal PT (2015) Antimicrobial, antioxidant, cytotoxic and molecular docking properties of $\mathrm{N}$ - benzyl-2,2,2trifluoroacetamide. Appl Nanosci 5:207-216

Bhadury P, Mohammad BT, Wright PC (2006) The current status of natural products from marine fungi and their potential as anti-infective agents. J Ind Microbiol Biotechnol 33:325-337

Choi JS, Park NH, Hwang SY, Sohn JH, Cho KK, Choi IS (2013) The antibacterial activity of various saturated and unsaturated fatty acids against several oral pathogens. J Environ Biol 34(4):673-676

Collins CH, Lyne PM (1985) Microbiological methods, 5th edition. Butterworth \& Co. Pub. Ltd, London \& Toronto, pp 167-181

Cueto M, Jensen PR, Kauffman C, Fenical W, Lobkovsky E, Clardy J (2001) Pestalone, a new antibiotic produced by a marine fungus in response to bacterial challenge. J Nat Prod 64:1444-1446

Donia M, Hamann MT (2003) Marine natural products and their potential applications as anti-infective agents. Lancet Infect Dis 3:338-348

Dulay RMR, Cabalar AC, De Roxas MJB, Concepcion JMP, Esmeralda M, Jimenez N, Aguilar JC, De Guzman EJ, Cruz NE, Santiago JQ, Samoy JR, Bustillos RG, Kalaw SP, RGP R (2015b) Proximate composition and antioxidant activity of Panaeolus antillarium, a wild coprophilous mushroom. Curr Res Environ Appl Mycol 5(1):52-59

Dulay RMR, Flores KS, Tiniola RC, Marquez DHH, Cruz AGD, Kalaw SP, Reyes RG (2015a) Mycelial biomass production and antioxidant activity of Lentinus tigrinus and Lentinus sajor-caju in indigenous liquid culture. Mycosphere 6(6):659-666

El-Neekety AA, Abdel-Aziz MS, Hathout AS, Hamed AA (2016) Molecular identification of newly isolated non-toxigenic fungal strains having antiaflatoxigenic, antimicrobial and antioxidant activities. Der Pharma Chemica 8(20):121-134

El-Shafei H, Abdel-Aziz M, Ghaly M, Abdalla A (2010) Optimizing some factors affecting alkaline protease production by a marine bacterium Streptomyces albidoflavus. In: Proceeding of Fifth Scientific Environmental Conference. Zagazig University, pp 125-142

Haefner B (2003) Drugs from the deep: marine natural products as drug candidates. Drug Discov Today 8:536-544

Kjer J, Debbab A, Aly AH, Proksch P (2010) Methods for isolation of marine-derived endophytic fungi and their bioactive secondary products. Nat Protoc 5:479-490

Mathan S, Subramanian V, Nagamony S (2013) Optimization and antimicrobial metabolite production from endophytic fungus Aspergillus terreus KC 582297. Eur J Exp Bio 3(4):138-144

Matsushige K, Basnet P, Kadota S, Namba T (1996) Potent free radical scavenging activity of dicaffeoylquinic acid derivatives from propolis. J Trad Med 13:217-228

Miao L, Kwong TFN, Qian P-Y (2006) Effect of culture conditions on mycelial growth, antibacterial activity, and metabolite profiles of the marine-derived fungus Arthrinium c.f. saccharicola. Appl Microbiol Biotechnol 72:1063-1073

Moulisha B, Bikash MN, Partha P, Kumar GA, Sukdeb B, Kanti HP (2009) In vitro anti-leishmanial and anti-tumour activities of a pentacyclic triterpenoid compound isolated from the fruits of Dregea volubilis Benth Asclepiadaceae. Trop J Pharm Res 8:127-131

Noaman NH, Abdel Fattah M, Khaleafa M, Zaky SH (2004) Factors affecting antimicrobial activity of Synechococcus leopoliensis. Microbiol Res 159:395-402

Park SH, Kwon KK, Lee D-S, Lee HK (2002) Morphological diversity of marine microorganisms on different isolation media. J Microbiol 40:161-165

Pomponi, S.A., 1999. The bioprocess-technological potential of the sea. J. Biotechnol., 70: 5-13

Saleem M, Ali MS, Hussain S, Jabbar A, Ashraf M, Lee YS (2007) Marine natural products of fungal origin. Nat Prod Rep 24:1142-1152

Talia JM, Debattista NB, Pappano NB (2011) New antimicrobial combinations: substituted chalcones-oxacillin against methicillin resistant Staphylococcus aureus. Braz J Microbiol 42(2):470-475 
Youssef AM, Abdel-Aziz MS, El-Sayed SM (2014) Chitosan nanocomposite films based on Ag-NP and Au-NP biosynthesis by Bacillus subtilis as packaging materials. Int J Biol Macromol 69:185-191

Zainuddin N, Alias SA, Lee CW, Ebel R, Othman NA, Awang K, Mukhtar MR (2010) Antimicrobial activities of marine fungi from Malaysia. Bot Mar 53:507-513

Zheng CJ, Yoo JS, Lee TG, Cho HY, Kim YH, Kim WG (2005) Fatty acid synthesis is a target for antibacterial activity of unsaturated fatty acids. FEBS Lett 579: 5157-5162

Zhou R, Liao X, Li H, Li J, Feng P, Zhao B, Xu S (2018) Isolation and synthesis of misszrtine $A$ : a novel indole alkaloid from marine sponge-associated Aspergillus sp. SCSIO XWS03F03. Front Chem 6:212 Epub 2018 Jun 13

Submit your manuscript to a SpringerOpen ${ }^{\odot}$ journal and benefit from:

- Convenient online submission

- Rigorous peer review

- Open access: articles freely available online

- High visibility within the field

- Retaining the copyright to your article

Submit your next manuscript at $\boldsymbol{\nabla}$ springeropen.com 